\title{
ON A UNIFORM ASYMPTOTIC EXPANSION OF A FOURIER-TYPE INTEGRAL*
}

\author{
$\mathrm{BY}$ \\ R. WONG \\ University of Manitoba, Winnipeg
}

\begin{abstract}
An alternative derivation is given for a uniform asymptotic expansion of the integral

$$
I(h)=\int_{q}^{z} g\left(\sqrt{y^{2}-q^{2}}\right) \sin y h d y \quad(h \rightarrow+\infty),
$$

which has been obtained recently by Schmidt. Here $q$ varies in the interval $\left[0, q_{0}\right], q_{0}$ is some fixed number less than $z$, and $z$ is finite. A similar result is obtained for integrals having an infinite range of integration. Realistic bounds are also provided for the error terms associated with the expansions. Our approach is based on a summability method introduced by Olver.
\end{abstract}

1. Introduction. In a recent paper [9], Schmidt has investigated the asymptotic behavior of the Fourier integral

$$
I(h)=\int_{q}^{z} g\left(\sqrt{y^{2}-q^{2}}\right) \sin y h d y,
$$

where $q$ and $z$ are nonnegative and $h$ is a large real parameter. The function $g(x)$ was assumed to be of the form $g(x)=f(x) / x$ with $f(x)$ being $n+1$ times continuously differentiable in $0 \leq x \leq\left(z^{2}-q^{2}\right)^{1 / 2}$ and $2 n$ times continuously differentiable near the origin. According to Schmidt, integrals of the type (1.1) occur in the calculation of the smallangle $x$-ray scattering from membranes; see [8] and [11].

For fixed $q$, asymptotic expansion of $I(h)$ can be obtained by either the integration by parts technique [3, Sec. 2.8] or the method based on summability [7]. However, the asymptotic expansion thus obtained for the case $q>0$ does not tend, as $q \rightarrow 0^{+}$, to the expansion for $q=0$; i.e., the expansion is not uniformly valid for $q$ arbitrarily close to 0 . This lack of uniformity is due to the possible coalescence of two critical points: the branch point singularities at $y=q$ and $y=-q$, one at an endpoint and one outside of the interval of integration. A similar problem, in which an exterior branch point coalesces with an endpoint, was considered earlier by Erdélyi [4].

In [9], Schmidt derives an asymptotic expansion that holds uniformly for $0 \leq q \leq q_{0}$,

\footnotetext{
* Received September 4, 1979. This research was partially supported by the National Science and Engineering Research Council of Canada under Contract A7359.
} 
where $q_{0}$ is some fixed number less than $z$ and $z$ is finite. His method is based on an integration by parts procedure, and resembles that of Bleistein [1] in the treatment of an algebraic singularity near a stationary point. Schmidt also provides an explicit expression for the error term associated with the expansion. However, the error term given in [9, Eqs. (6)-(8)] involves Bessel functions, and appears to be complicated and difficult to estimate.

The purpose of this paper is to present an alternative (and, it is hoped, better motivated) derivation of the expansion given by Schmidt, and to extend the result to the case of an infinite range of integration. The error terms in our expansions involve only the sine function, and realistic bounds are also provided for them. Our approach is based on a summability method recently introduced by Olver [7] and subsequently extended by the present author [10].

In Sec. 2, we list and discuss the basic assumptions. The main results are stated in Sec. 3. In Sec. 4, we give a preliminary lemma. The proofs of the main results are given in Secs. 5 and 6. Error bounds for the asymptotic expansions are derived in Sec. 7; this section also contains some illustrative examples.

2. Assumptions. Throughout this paper, we shall assume that the following conditions hold. These conditions are weaker than the ones imposed by Schmidt [9], and are similar to those adopted by Olver for stationary phase approximations [7]. integer.

$\left(\mathrm{A}_{1}\right) g^{(n)}(x)$ is continuous in the interval $0<x<\left(z^{2}-q^{2}\right)^{1 / 2}$, where $n$ is a positive

$\left(\mathrm{A}_{2}\right)$ As $x \rightarrow 0^{+}$,

$$
g(x) \sim \sum_{s=0}^{\infty} a_{s} x^{s+x-1}, \quad 0 \leq \alpha<1,
$$

and the asymptotic expansions of the derivatives of $g$, up to and including order $n$, can be obtained by differentiating this series term by term.

$\left(\mathrm{A}_{3}\right)$ When $z$ is finite, each of the functions

$$
G^{(s)}(y)=\frac{d^{s}}{d y^{s}} g\left(\sqrt{y^{2}-q^{2}}\right), \quad s=0,1, \ldots, n
$$

tends to a finite limit, denoted by $G^{(s)}\left(z^{-}\right)$, as $y \rightarrow z^{-}$.

$\left(\mathrm{A}_{4}\right)$ When $z=\infty$, each of the integrals

$$
\int_{c}^{\infty} G^{(s)}(y) \sin \left(\frac{s \pi}{2}+h y\right) d y, \quad s=0,1, \ldots, n
$$

converges uniformly for all sufficiently large $h$, where $c$ is some fixed positive number.

Remarks. (i) If $g(x)=f(x) / x$ and $f(x)$ is $2 n$ times continuously differentiable at $x=0$ then it is easy to see that

$$
g(x)=\sum_{j=0}^{2 n-1} \frac{f^{(j)}(0)}{j !} x^{j-1}+O\left(x^{2 n-1}\right) \quad \text { as } \quad x \rightarrow 0^{+} .
$$

Furthermore, if $f(x)$ is $n$ times continuously differentiable in $\left[0,\left(z^{2}-q^{2}\right)^{1 / 2}\right]$, the expansion (2.3) can be differentiated $n$ times. Thus, our assumptions $\left(\mathrm{A}_{1}\right),\left(\mathrm{A}_{2}\right)$ and $\left(\mathrm{A}_{3}\right)$ are all met by the conditions of Schmidt [9]. (Schmidt, in fact, assumes that $f(x)$ is $n+1$ times 
continuously differentiable in $\left[0,\left(z^{2}-q^{2}\right)^{1 / 2}\right]$.) (ii) In (2.1), the condition $\alpha<1$ is not really a restriction, since an appropriate number of leading coefficients $a_{s}$ may vanish. (iii) Assumption $\left(\mathrm{A}_{4}\right)$, with $s=0$, implies that in the case of an infinite range of integration the original integral $I(h)$ in $(1.1)$ converges uniformly. Moreover, by integration by parts,

$$
\begin{aligned}
\int G^{(s)}(y) \sin \left(\frac{s \pi}{2}+h y\right) d y= & -\cos \left(\frac{s \pi}{2}+h y\right) \frac{G^{(s)}(y)}{h} \\
& +\frac{1}{h} \int G^{(s+1)}(y) \sin \left(\frac{s+1}{2} \pi+h y\right) d y
\end{aligned}
$$

for $0 \leq s \leq n-1$. Thus, a necessary and sufficient condition for the uniform convergence of the integrals in $\left(\mathrm{A}_{4}\right)$ is that $I(h)$ converge uniformly at its upper limit and

$$
G^{(s)}(y) \rightarrow 0 \quad \text { as } y \rightarrow \infty, \quad s=0,1, \ldots, n-1 .
$$

3. Statement of results. Throughout this paper, we shall adhere to the notation set in the preceding and the present sections. For each $n \geq 0$, we put

$$
G(y)=G_{2 n}(y)+R_{2 n}(y),
$$

where $G(y)$ is as given in (2.2) and $G_{2 n}(y)$ denotes the sum

$$
G_{2 n}(y)=\sum_{s=0}^{2 n-1} a_{s}\left(y^{2}-q^{2}\right)^{(s+\alpha-1) / 2} .
$$

(As usual, empty sums are understood to be zero.) Note that from $\left(A_{2}\right)$ we have

$$
R_{2 n}^{(n)}(y)=0\left\{(y-q)^{(\alpha-1) / 2}\right\}, \quad \text { as } \quad y \rightarrow q^{+},
$$

and that

$$
G_{2 n}^{(2 n)}(y)=O\left(y^{-2+\alpha}\right), \quad \text { as } \quad y \rightarrow+\infty .
$$

These order estimates ensure that all the integrals occurring in the following statements converge.

THREOREM 1. If $z$ is finite then under the assumptions $\left(A_{1}\right),\left(A_{2}\right)$ and $\left(A_{3}\right)$ we have

$$
\begin{aligned}
I(h)= & \sum_{s=0}^{2 n-1} J_{-(s+\alpha) / 2}(q h) \frac{a_{s}(q)}{h^{(s+\alpha) / 2}}+\delta_{n}(h) \\
& -\sum_{s=0}^{n-1} \sin \left(\frac{s+1}{2} \pi+h z\right) \frac{G^{(s)}\left(z^{-}\right)}{h^{s+1}}-\varepsilon_{n}(h),
\end{aligned}
$$

where

$$
a_{s}(q)=\frac{\sqrt{ } \pi}{2} a_{s} \Gamma\left(\frac{s+\alpha+1}{2}\right)(2 q)^{(s+\alpha) / 2}
$$

and the error terms are given by

$$
\delta_{n}(h)=\frac{1}{h^{n}} \int_{q}^{z} R_{2 n}^{(n)}(y) \sin \left(\frac{n \pi}{2}+h y\right) d y
$$


and

$$
\varepsilon_{n}(h)=\sum_{s=n}^{2 n-1} \sin \left(\frac{s+1}{2} \pi+h z\right) \frac{G_{2 n}^{(\mathrm{s})}(z)}{h^{s+1}}+\frac{(-1)^{n}}{h^{2 n}} \int_{z}^{\infty} G_{2 n}^{(2 n)}(y) \sin y h d y .
$$

THEOREM 2. If $z$ is infinite then under the assumptions $\left(A_{1}\right),\left(A_{2}\right)$ and $\left(A_{4}\right)$ we have

$$
I(h)=\sum_{s=0}^{2 n-1} J_{-(s+\alpha) / 2}(q h) \frac{a_{s}(q)}{h^{(s+\alpha) / 2}}+\delta_{n}(h)-\varepsilon_{n}(h),
$$

where the coefficients $a_{s}(q)$ have the same meaning as given in (3.6) and the remainders satisfy

$$
\delta_{n}(h)=\frac{1}{h^{n}}\left[\int_{q}^{a} R_{2 n}^{(n)}(y) \sin \left(\frac{n \pi}{2}+h y\right) d y+\int_{a}^{\infty} G^{(n)}(y) \sin \left(\frac{n \pi}{2}+h y\right) d y\right]
$$

and

$$
\varepsilon_{n}(h)=\sum_{s=n}^{2 n-1} \sin \left(\frac{s+1}{2} \pi+h a\right) \frac{G_{2 n}^{(s)}(a)}{h^{s+1}}+\frac{(-1)^{n}}{h^{2 n}} \int_{a}^{\infty} G_{2 n}^{(2 n)}(y) \sin y h d y,
$$

$a$ being an arbitrary positive number $>q$.

In view of (2.5) and (3.4), one naturally expects that the result (3.9) should follow immediately from (3.5) by letting $z \rightarrow+\infty$. However, this is not the case, since the integral (3.7) may diverge if we replace $z$ by $+\infty$ and the terms involving $G_{2 n}^{(s)}(z), s=n$, $\ldots, 2 n-1$, in (3.8) may not all tend to zero as $z \rightarrow \infty$. Nevertheless, if both of these conditions happen to be true then the above remark applies and the expression for the error may be considerably simplified; see Example 2 in Sec. 7.

On account of the assumptions $\left(\mathrm{A}_{3}\right)$ and $\left(\mathrm{A}_{4}\right)$ with $s=n$ and the estimate (3.3), it is easy to see that the error terms $\delta_{n}(h)$ given in (3.7) and (3.10) both satisfy

$$
\delta_{n}(h)=o\left(h^{-n}\right) \quad \text { as } \quad h \rightarrow \infty,
$$

by the Riemann-Lebesgue lemma [6, p. 73]. The other error terms $\varepsilon_{n}(h)$ given in (3.8) and (3.11) clearly also satisfy the above order relation. In fact, we have

$$
\varepsilon_{n}(h)=O\left(h^{-n-1}\right) \quad \text { as } h \rightarrow \infty .
$$

Moreover, both results (3.12) and (3.13) hold uniformly for $0 \leq q \leq q_{0}$. This confirms the uniformly asymptotic nature of the expansions (3.5) and (3.9), and hence we may write, as $h \rightarrow+\infty$,

$$
\begin{aligned}
I(h) \sim & \sum_{s=0}^{\infty} J_{-(s+x) / 2}(q h) \frac{a_{s}(q)}{h^{(s+x) / 2}} \\
& -\sum_{s=0}^{\infty} \sin \left(\frac{s+1}{2} \pi+h z\right) \frac{G^{(s)}\left(z^{-}\right)}{h^{s+1}}
\end{aligned}
$$

if $z$ is finite, and

$$
I(h) \sim \sum_{s=0}^{\infty} J_{-(s+x) / 2}(q h) \frac{a_{s}(q)}{h^{(s+x) / 2}}
$$

if $z$ is infinite, both uniformly in $q$ for $0 \leq q \leq q_{0}$. 
4. Preliminary lemmas. It is well known that

$$
\int_{1}^{\infty}\left(t^{2}-1\right)^{\lambda} \sin x t d t=\frac{\sqrt{ } \pi \Gamma(\lambda+1)}{2\left(\frac{1}{2} x\right)^{\lambda+1 / 2}} J_{-\lambda-1 / 2}(x)
$$

for $-1<\lambda<0$ and $x>0$; see, for example, [2, p. 81] and [6, p. 244]. The central idea in this paper is to observe that if the integral in (4.1) is interpreted in the sense of Abel summability [5, p. 11, Eq. (1.5.3)] then the above identity holds for all $\lambda>-1$ and that the expansion (3.15) can be obtained by formally integrating the series (2.1) term by term.

Lemma 1 . When $x>0$ and $\lambda>-1$,

$$
\lim _{\eta \rightarrow 0^{+}} \int_{1}^{\infty} e^{-\eta t}\left(t^{2}-1\right)^{\lambda} \sin x t d t=\frac{\sqrt{ } \pi \Gamma(\lambda+1)}{2\left(\frac{1}{2} x\right)^{\lambda+1 / 2}} J_{-\lambda-1 / 2}(x) .
$$

Proof. An integral representation corresponding to (4.1) for the modified Bessel function $K_{v}$ is given by

$$
\int_{1}^{\infty}\left(t^{2}-1\right)^{\lambda} e^{-z t} d t=\frac{\Gamma(\lambda+1)}{\sqrt{\pi\left(\frac{1}{2} z\right)^{\lambda+1 / 2}}} K_{-\lambda-1 / 2}(z)
$$

where $\operatorname{Re} z>0$ and $\lambda>-1$; see [2, p. 82]. Thus, replacing $\sin x t$ by $\left(e^{i x t}-e^{-i x t}\right) / 2 i$, we have

$$
\int_{1}^{\infty} e^{-\eta t}\left(t^{2}-1\right) \sin x t d t=\frac{\Gamma(\lambda+1) 2^{\lambda+1 / 2}}{2 i \sqrt{\pi}}\left[\frac{K_{-\lambda-1 / 2}(\eta-i x)}{(\eta-i x)^{\lambda+1 / 2}}-\frac{K_{-\lambda-1 / 2}(\eta+i x)}{(\eta+i x)^{\lambda+1 / 2}}\right] .
$$

Taking a limit on both sides gives

$$
\begin{aligned}
\lim _{\eta \rightarrow 0^{+}} \int_{1}^{\infty} e^{-\eta t}\left(t^{2}-1\right)^{\lambda} \sin x t d t= & \frac{\Gamma(\lambda+1) 2^{\lambda+1 / 2}}{2 i \sqrt{\pi} x^{\lambda+1 / 2}}\left[e^{\pi i(\lambda+1 / 2) 2} K_{-\lambda-1 / 2}\left(e^{-i \pi / 2} x\right)\right. \\
& \left.-e^{-\pi i(\lambda+1 / 2) / 2} K_{-\lambda-1 / 2}\left(e^{i \pi / 2} x\right)\right] .
\end{aligned}
$$

In view of the connection formulas [2, pp. 4 and 6$]$

$$
K_{v}\left(e^{\pi i / 2} x\right)=-\frac{\pi i}{2} e^{-i \pi v / 2} H_{v}^{(2)}(x), \quad K_{v}\left(e^{-\pi i / 2} x\right)=\frac{\pi i}{2} e^{v \pi i / 2} H_{v}^{(1)}(x)
$$

and

$$
J_{v}(x)=\frac{1}{2}\left[H_{v}^{(1)}(x)+H_{v}^{(2)}(x)\right],
$$

the right-hand side of (4.3) is equal to

$$
\frac{\sqrt{ } \pi \Gamma(\lambda+1)}{2\left(\frac{1}{2} x\right)^{\lambda+1 / 2}} J_{-\lambda-1 / 2}(x) .
$$

This completes the proof of the lemma.

For convenience, we also recall the following result, a proof of which can be found in [5, p. 151]. 
Lemma 2. If the integral $\int_{0}^{\infty} f(t) d t$ exists as an improper Riemann integral then

$$
\lim _{\varepsilon \rightarrow 0^{+}} \int_{0}^{\infty} e^{-\varepsilon t} f(t) d t=\int_{0}^{\infty} f(t) d t
$$

5. Proof of Theorem 1. From (3.1) and (3.2) we have

$$
\begin{aligned}
\int_{q}^{z} e^{-n y} G(y) \sin y h d y= & \sum_{s=0}^{2 n-1} a_{s} \int_{q}^{\infty} e^{-\eta y}\left(y^{2}-q^{2}\right)^{(s+x) / 2} \sin y h d y \\
& +E_{2 n}(\eta, h)-F_{2 n}(\eta, h)
\end{aligned}
$$

where

$$
E_{2 n}(\eta, h)=\int_{q}^{z} e^{-n y} R_{2 n}(y) \sin y h d y
$$

and

$$
F_{2 n}(\eta, h)=\int_{z}^{\infty} e^{-\eta y} G_{2 n}(y) \sin y h d y .
$$

Since $G(y)=g\left(\left(y^{2}-q^{2}\right)^{1 / 2}\right)$, by Lemmas 1 and 2 , we have, upon passing to the limit,

$$
I(h)=\sum_{s=0}^{2 n-1} J_{-(s+x) / 2}(q h) \frac{a_{s}(q)}{h^{(s+x) / 2}}+E_{2 n}(h)-F_{2 n}(h),
$$

where $a_{s}(q)$ is as given in (3.6) and

$$
E_{2 n}(h)=\lim _{\eta \rightarrow 0^{+}} E_{2 n}(\eta, h), \quad F_{2 n}(h)=\lim _{\eta \rightarrow 0^{+}} F_{2 n}(\eta, h) .
$$

In (5.2), we replace $\sin y h$ by $\left(e^{i y h}-e^{-i y h}\right) / 2 i$ and integrate by parts $n$ times. Since by $\left(\mathrm{A}_{2}\right)$

$$
R_{2 n}^{(s)}(y)=O\left\{(y-q)^{n-s+(x-1) / 2}\right\} \rightarrow 0 \quad \text { as } \quad y \rightarrow q^{+},
$$

for $s=0,1, \ldots, n-1$, the integrated terms all vanish at the lower limit of the integral. Taking the limit as $\eta \rightarrow 0^{+}$, we have from (5.5)

$$
E_{2 n}(h)=-\sum_{s=0}^{n-1} \sin \left(\frac{s+1}{2} \pi+h z\right) \frac{R_{2 n}^{(s)}\left(z^{-}\right)}{h^{s+1}}+\frac{1}{h^{n}} \int_{q}^{z} R_{2 n}^{(n)}(y) \sin \left(\frac{n \pi}{2}+h y\right) d y .
$$

Note that the last integral converges at the lower limit, on account of (3.3).

Now we consider $F_{2 n}(h)$. First, we proceed as above via partial integration of (5.3) $2 n$ times. In view of the exponential factor $e^{-\eta y}$, the integrated terms all vanish at the upper limit of the integral. Next, we take the limit as $\eta \rightarrow 0^{+}$. From (5.5) we have

$$
F_{2 n}(h)=\sum_{s=0}^{2 n-1} \sin \left(\frac{s+1}{2} \pi+h z\right) \frac{G_{2 n}^{(s)}(z)}{h^{s+1}}+\frac{(-1)^{n}}{h^{2 n}} \int_{z}^{\infty} G_{2 n}^{(2 n)}(y) \sin y h d y .
$$

Substituting (3.1) in (5.6) with $y=z^{-}$and subtracting (5.7) yields

$$
E_{2 n}(h)-F_{2 n}(h)=-\sum_{s=0}^{n-1} \sin \left(\frac{s+1}{2} \pi+h z\right) \frac{G^{(s)}\left(z^{-}\right)}{h^{s+1}}+\delta_{n}(h)-\varepsilon_{n}(h),
$$


where $\delta_{n}(h)$ and $\varepsilon_{n}(h)$ are as given in (3.7) and (3.8). Coupling (5.4) and (5.8), we have the desired result (3.5).

6. Proof of Theorem 2. In view of (2.5), we have, from Theorem 1,

$$
I(h)=\sum_{s=0}^{2 n-1} J_{-(s+x) / 2}(q h) \frac{a_{s}(q)}{h^{(s+\alpha) / 2}}+E_{2 n}^{*}(h),
$$

where

$$
\begin{aligned}
E_{2 n}^{*}(h)= & \lim _{z \rightarrow \infty}\left[-\sum_{s=n}^{2 n-1} \sin \left(\frac{s+1}{2} \pi+h z\right) \frac{G_{2 n}^{(s)}(z)}{h^{s+1}}\right. \\
& \left.+\frac{1}{h^{n}} \int_{q}^{z} R_{2 n}^{(n)}(y) \sin \left(\frac{n \pi}{2}+h y\right) d y\right] .
\end{aligned}
$$

Let $T$ denote the integral in (6.2). For any $a$ in the interval $(q, z)$, we may break the range of integration at $y=a$ so that $T=T_{1}+T_{2}$ with $T_{1}$ and $T_{2}$ corresponding, respectively, to the intervals $(q, a)$ and $(a, z)$. Note that $T_{1}$ agrees with the first integral on the right of (3.10). In $T_{2}$ we insert (3.1), and thus obtain $T_{2}=T_{21}+T_{22}$ with

$$
T_{22}=-\int_{a}^{z} G_{2 n}^{(n)}(y) \sin \left(\frac{n \pi}{2}+h y\right) d y .
$$

Repeated integration by parts shows that $T_{22}$ is equal to

$$
\begin{aligned}
\sum_{s=n}^{2 n-1} \sin \left(\frac{s+1}{2} \pi+h z\right) \frac{G_{2 n}^{(s)}(z)}{h^{s-n+1}}-\sum_{s=n}^{2 n-1} \sin \left(\frac{s+1}{2} \pi+h a\right) \frac{G_{2 n}^{(s)}(a)}{h^{s-n+1}} & \\
& -\frac{(-1)^{n}}{h^{n}} \int_{a}^{z} G_{2 n}^{(2 n)}(y) \sin y h d y .
\end{aligned}
$$

The first sum is identical with that in (6.2) except for a factor of $h^{-n}$. Hence, upon adding the contributions from $T_{1}, T_{21}$ and $T_{22}$, we have

$$
\begin{aligned}
E_{2 n}^{*}(h)= & \lim _{z \rightarrow \infty}\left\{\frac{1}{h^{n}} \int_{q}^{a} R_{2 n}^{(n)}(y) \sin \left(\frac{n \pi}{2}+h y\right) d y+\int_{a}^{z} G^{(n)}(y) \sin \left(\frac{n \pi}{2}+h y\right) d y\right. \\
& \left.-\sum_{s=n}^{2 n-1} \sin \left(\frac{s+1}{2} \pi+h a\right) \frac{G_{2 n}^{(s)}(a)}{h^{s+1}}-\frac{(-1)^{n}}{h^{2 n}} \int_{a}^{z} G_{2 n}^{(2 n)}(y) \sin y h d y\right\} .
\end{aligned}
$$

The first and the third terms on the right are independent of $z$, and the second and fourth terms converge as $z \rightarrow+\infty$. Therefore,

$$
E_{2 n}^{*}(h)=\delta_{n}(h)-\varepsilon_{n}(h),
$$

where $\delta_{n}(h)$ and $\varepsilon_{n}(h)$ are as given in (3.10) and (3.11). This, together with (6.1), proves the result stated in Theorem 2 .

7. Error bounds and examples. Without loss of generality, we may assume that $h \geq 1$. From (3.7) and (3.8), we have

$$
\left|\delta_{n}(h)\right| \leq \frac{1}{h^{n}} \int_{q}^{z}\left|R_{2 n}^{(n)}(y)\right| d y
$$


and

$$
\left|\varepsilon_{n}(h)\right| \leq \frac{1}{h^{n+1}}\left[\sum_{s=n}^{2 n-1}\left|G_{2 n}^{(s)}(z)\right|+\int_{z}^{\infty}\left|G_{2 n}^{(2 n)}(y)\right| d y\right]
$$

if $z$ is finite.

When $z$ is infinite, we use (3.10) and (3.11). Thus,

$$
\left|\delta_{n}(h)\right| \leq \frac{1}{h^{n}}\left[\int_{q}^{a}\left|R_{2 n}^{(n)}(y)\right| d y+\int_{a}^{\infty}\left|G^{(n)}(y)\right| d y\right]
$$

and

$$
\left.\left|\varepsilon_{n}(h)\right| \leq \frac{1}{h^{n+1}}\left[\sum_{s=n}^{2 n-1}\left|G_{2 n}^{(s)}(a)\right|+\int_{a}^{\infty} \mid G_{2 n}^{(2 n}\right)(y) \mid d y\right] .
$$

Note that the integrals in (7.1), (7.2) and (7.4) all converge under the conditions of Theorems 1 and 2. However, the convergence of the integral in (7.3) is not guaranteed by these assumptions.

An examination of the asymptotic behavior of the Bessel function $J_{v}(t)$, in the two limits $t \rightarrow+\infty$ and $t \rightarrow 0^{+}$, reveals that the above error bounds are extremely crude. Since $J_{v}(t) \sim(2 / \pi t)^{1 / 2} \cos \left(t-\frac{1}{2} v \pi-\frac{1}{4} \pi\right)$ as $t \rightarrow+\infty$ and $J_{v}(t) \sim\left(\frac{1}{2} t\right)^{v} / \Gamma(v+1)$ as $t \rightarrow 0^{+}$, the errors in the asymptotic approximations (3.5) and (3.9) should be $O\left(h^{-n-(x+1) / 2}\right)$ if $q h$ is large and $O\left(h^{-2 n-x}\right)$ if $q h$ is small. Thus, the estimates in (7.1)-(7.4) fall short of the actual result. However, it is extremely difficult to get strict error bounds for asymptotic expansions of the above type. Sometimes we will have to be content with a bound involving a lower power of the asymptotic variable than that known to be obtainable from the big $O$-estimates. One way to arrive at a bound involving the correct power of the asymptotic variable is to continue the expansion to a few more terms, and then use these terms together with the bound for the new remainder term (which is of a lower asymptotic order of magnitude).

Example 1. Let $g(x)=e^{i x} / x$ and consider

$$
I(h)=\int_{q}^{z} g\left(\sqrt{y^{2}-q^{2}}\right) \sin y h d y,
$$

where $0 \leq q \leq q_{0}<z<\infty$. For all real $x \neq 0$,

$$
g(x)=\sum_{s=0}^{\infty} \frac{i^{s}}{s !} x^{s-1}
$$

Thus, in (2.1), $\alpha=0$ and $a_{s}=i s / s$ !. To derive the dominant term of the uniform asymptotic expansion of $I(h)$, we take $n=1$ in (3.5), and put $g_{2}(x)=(1 / x)+i$ and $r_{2}(x)=g(x)-$ $g_{2}(x)$. Then, in the notation of (3.1) and (3.2), we have

$$
G_{2}(y)=g_{2}(x) \quad \text { and } \quad R_{2}(y)=r_{2}(x),
$$

where $x=\left(y^{2}-q^{2}\right)^{1 / 2}$. A simple calculation shows that

$$
G_{2}^{\prime}(y)=g_{2}^{\prime}(x) \frac{d x}{d y}=-\frac{y}{\left(y^{2}-q^{2}\right)^{3 / 2}}
$$


and

$$
\left|R_{2}^{\prime}(y)\right| \leq \frac{3}{2}\left(y / \sqrt{y^{2}-q^{2}}\right) .
$$

From (3.5) it now follows that

$$
\begin{aligned}
& I(h)=\frac{\pi}{2} J_{0}(q h)+\frac{i}{h} \cos q h+\delta_{1}(h) \\
& -\frac{\exp \left(i \sqrt{z^{2}-q^{2}}\right)}{\sqrt{z^{2}-q^{2}}} \cdot \frac{\cos h z}{h}-\varepsilon_{1}(h) .
\end{aligned}
$$

(Note: $J_{-1 / 2}(t)=(2 / \pi t)^{1 / 2} \cos t$.) The bounds (7.1) and (7.7) yield

$$
\left|\delta_{1}(h)\right| \leq \frac{3}{2 h} \sqrt{z^{2}-q^{2}}
$$

Also, from (7.2) and (7.6) we derive

$$
\left|\varepsilon_{1}(h)\right| \leq \frac{1}{h^{2}}\left[\frac{z}{\left(z^{2}-q^{2}\right)^{3 / 2}}+\int_{z}^{\infty} \frac{2 y^{2}+q^{2}}{\left(y^{2}-q^{2}\right)^{5 / 2}} d y\right] .
$$

The last integral can be evaluated explicitly, and we have

$$
\left|\varepsilon_{1}(h)\right| \leq \frac{2 q^{2}}{z\left(z^{2}-q^{2}\right)^{3 / 2}} \cdot \frac{1}{h^{2}} .
$$

A combination of the results (7.8), (7.9) and (7.10) then gives

with the strict error bound

$$
I(h)=\frac{\pi}{2} J_{0}(q h)+\rho_{1}(h)
$$

$$
\left|\rho_{1}(h)\right| \leq \frac{1}{h}\left[1+\frac{3}{2} \sqrt{z^{2}-q^{2}}+\frac{1}{\sqrt{z^{2}-q^{2}}}+\frac{2 q^{2}}{z\left(z^{2}-q^{2}\right)^{3 / 2}}\right] .
$$

Note that in order to obtain an error estimate for the one-term approximation in (7.11), we have taken three terms in the asymptotic expansion of $I(h)$.

Example 2. Let $g(x)=1 / x(x+1)$ and consider

$$
I(h)=\int_{q}^{\infty} g\left(\sqrt{y^{2}-q^{2}}\right) \sin y h d y,
$$

where $0 \leq q \leq q_{0}<+\infty$. For $0<x<1$,

$$
g(x)=\sum_{s=0}^{\infty}(-1)^{s} x^{s-1}
$$

Thus, with $g_{2}(x)=1 / x-1$ and $r_{2}(x)=g(x)-g_{2}(x)$, we have

$$
G_{2}(y)=g_{2}(x) \quad \text { and } \quad R_{2}(y)=r_{2}(x)
$$

where $x=\left(y^{2}-q^{2}\right)^{1 / 2}$. Since $\alpha=0$ and $a_{s}=(-1)^{s}$, it follows from (3.9) that

$$
I(h)=\frac{\pi}{2} J_{0}(q h)-\frac{1}{h} \cos q h+\delta_{1}(h)-\varepsilon_{1}(h)
$$


where, by (6.2) and (6.4),

$$
\delta_{1}(h)-\varepsilon_{1}(h)=\lim _{z \rightarrow \infty}\left[\frac{1}{h} \int_{q}^{z} R_{2}^{\prime}(y) \sin \left(\frac{\pi}{2}+h y\right) d y-\sin (\pi+h z) \frac{G_{2}^{\prime}(z)}{h^{2}}\right] .
$$

Since $G_{2}^{\prime}(z) \rightarrow 0$ as $z \rightarrow+\infty$ and

$$
\int_{q}^{\infty} R_{2}^{\prime}(y) d y=\int_{0}^{\infty} \frac{1}{(1+x)^{2}} d x=1
$$

by (7.14), we have from (7.16)

$$
\delta_{1}(h)-\varepsilon_{1}(h)=\frac{1}{h} \int_{q}^{\infty} R_{2}^{\prime}(y) \cos y h d y=o\left(h^{-1}\right),
$$

as $h \rightarrow 0^{+}$. This expression is considerably simpler than that given in (3.10)-(3.11) with $n=1$. However, such a simple expression is not always available; see the remark following Theorem 2 .

\section{REFERENCES}

[1] N. Bleistein, Uniform asymptotic expansions of integrals with stationary point near algebraic singularity, Comm. Pure Appl. Math. 19, 353-370 (1966)

[2] A. Erdélyi, W. Magnus, F. Oberhettinger and F. Tricomi, Higher transcendental functions, Vol. 2, McGraw-Hill, New York, 1953

[3] A. Erdélyi, Asymptotic expansions, Dover, New York, 1956

[4] A. Erdélyi, Asymptotic evaluation of integrals involving a fractional derivative, SIAM J. Math. Anal. 5, 159-171 (1974)

[5] G. H. Hardy, Divergent series, Oxford University Press (Clarendon), London, 1949

[6] F. W. J. Olver, Asymptotics and special functions, Academic Press, New York (1974)

[7] F. W. J. Olver, Error bounds for stationary phase approximations, SIAM J. Math. Anal. 5, 19-29 (1974)

[8] P. W. Schmidt, Small-angle x-ray scattering from rods and platelets, J. Math. Phys. 7, 1295-1300 (1966)

[9] P. W. Schmidt, An asymptotic approximation for a type of Fourier integral, Math. Comp. 32, 1171-1182 (1978)

[10] R. Wong, Error bounds for asymptotic expansions of Hankel transforms, SIAM J. Math. Anal. 7, 799-808 (1976)

[11] H. Wu and P. W. Schmidt, to be published 